\title{
Research Status and Prospect Of Micro-polluted Water Pretreatment With Biological Aerated Filter
}

\author{
Qianqian Song ${ }^{1, a}$, Liping Qiu ${ }^{1, b^{*}}$, Shoubin Zhang ${ }^{1, c}$, Jiabin Wang ${ }^{1, d}$, Kang \\ $X_{i e}^{1, e}$
}

\author{
${ }^{1}$ School of Civil Engineering and Architecture, University of Jinan, No.336. West Road of Nan \\ Xinzhuang. Jinan. 250022, PR China \\ asdssyxxsqq@163.com, ${ }^{\text {b }}$ cea_qiulp@ujn.edu.cn, ${ }^{\mathrm{c}} \mathrm{cea}$ zhangsb@ujn.edu.cn, \\ dcea_wangjb@ujn.edu.cn, ${ }^{\mathrm{e}} \mathrm{cea}$ _xiek@ujn.edu.cn,
}

* Corresponding Author

\begin{abstract}
Keywords: Micro-polluted Water ; BAF; Biological Pre-treatment
Abstract. Recently, with the rapid development of industry, the pollution of organic compounds and ammonia nitrogen is serious in source water. It is difficult to ensure the removal efficiency of ammonia nitrogen and organic compounds in effluent by conventional treatment technology. The article brings out the treatment of micro-polluted water by biological aerated filter. This article discuss the pre-treatment of micro-polluted water with biological aerated filter from following aspects:the principle of micro-polluted water's treatment by BAF, the advantages of pollutant removal , and influencing factors of stable operation of BAF .In the end, this article introduces the practical application of the pre-treatment of micro-polluted water by biological aerated filter, and makes prospects for its development and application.
\end{abstract}

\section{Introduction}

In recent years, with the rapid development of industry, accelerated urbanization process and sharp increase in the use of agricultural chemicals, drinking water were polluted in different degree. Many rivers, groundwater and other water sources lose the function of being drinking water. But in the situation of shortage of water resources, micro-polluted water is still an important source of water. The species and quantity of organic compounds surge. And the phenomenon of eutrophication is aggravated. With the progress of science and technology, technology of analysis of water quality and the national drinking water standards is improving. The removal efficiency of dissolved organic compounds, ammonia nitrogen, iron and manganese is not ideal by using conventional water treatment. Addition of chemical reagents is easy to produce by-products. Aiming to solve this phenomenon, many scholars studied biological pre-treatment of micro-polluted water. With the help of the metabolism of the microbial population, the biological pre-treatment remove organic compounds, ammonia nitrogen, iron, manganese and other pollutants, and achieved considerable results. The removal rate of ammonia nitrogen reached more than $80 \%$. And turbidity, chroma, iron, manganese and other pollutants are better removed ${ }^{[1]}$.

\section{The principle of micro-polluted water's treatment by BAF}

The treatment medium of biological aerated filter is mainly the filter material of the reactor and the biofilm attached to the filter material. Pollutants were removed mainly through the filtration, adsorption and retention of filter material and biofilm, as well as the oxidation and decomposition of microorganism on biofilm. When the raw water containing the matrix passing through the filter material, the microorganism absorb nutrients in raw water. Microbes proliferate in the filler surface, after a certain period of time to form a biofilm. Mature biofilm surface contact with water, easy to absorb nutrients and dissolved oxygen, formed aerobic layer composed of aerobic and facultative microorganisms. Simultaneously, biofilm interior form anaerobic layer composed with Anaerobic and concurrent microorganisms ${ }^{[2]}$. At the same time, the proliferation of microorganisms and the accumulation of suspended substances, resulting in a gradual increase in the thickness of the biofilm, and the changes of biofilm structure. With the anaerobic layer aging, biofilm combined with filter 
material loosening. Under the action of shear and back flushing of the water, the aging biofilm will fall off ${ }^{[3]}$. After falling off the biofilm, a new biofilm will grow up, which will be carried out repeatedly.

\section{The advantages of pollutant removal}

The Removal of Turbidity. Organic pollutants concentration is relatively low in micro-polluted water. Oligotrophic bacteria have a great advantage in nutrient competition. Therefore biofilm to with oligotrophic bacteria has larger specific surface area and have stronger adsorption and biological effects for suspended particles. According to microscope observation, the filter surface covered dough, flocculent biofilm. And across biofilm, there are still more biofilm covering the surface of filter ${ }^{[4]}$. Thus, the surface of the filter material can still play a role in the adsorption and retention of suspended particles in water. In addition, vorticellidae and shield worm on biofilm, feed with free bacteria and the sludge particle, also helped to reduce the turbidity ${ }^{[4]}$. Aging biofilms in the settlement process, can also contribute to the settling of fine suspended particles in water.

The Removal of Organic Compounds. The removal of organic compounds is mainly through the following aspects (1)the degradation of small molecule organic compounds by microorganisms;(2)the decomposition of macromolecular organic compounds by microbial extracellular enzymes; (3)biological adsorption flocculation. Biofilm with larger specific surface area, adsorb some organic compounds .Meanwhile, microbial extracellular enzymes, containing polysaccharide and other viscous substances, have the effect of chemical flocculation, and have strong adsorption and flocculation for organic compounds. Liu et al used bamboo charcoal as filler of BAF in treatment effect micro-polluted water, and found that the average removal rate of $\mathrm{COD}_{\mathrm{Mn}}$ and ammonia nitrogen was $51.6 \%$ and $83.6 \%{ }^{[5]}$.

The Removal of Ammonia Nitrogen. The concentration of organic matrix, ammonia nitrogen and nitrite nitrogen in micro-polluted water, is lower relative to the corresponding substances in the sewage. Therefore, the oligotrophic bacteria have advantages in nutrient competition. Oligotrophic Bacteria has larger specific surface area, for matrix which it can make use of, has a strong affinity. The breathing rate of oligotrophic microorganism is low. Oligotrophic microorganism have relatively smaller maximum proliferation rate and Monod half rate constant. So it can adapt to low nutrient environments. Removal of ammonia nitrogen in micro-polluted water by biological method, achieve through the cultivation of oligotrophic microorganism such as the nitrite bacteria and nitrate bacteria ${ }^{[6]}$. Ammonia nitrogen under the action of nitrite bacteria transform into nitrite, and then in under the action of nitrifying bacteria into nitrate. The generation time of nitrifying bacteria is relatively long. Microorganism growth is immobilized in BAF. So microbial in the reactor can get a longer residence time, Nitrite and nitrification bacteria have enough time to accumulate. Therefore the removal of ammonia by BAF has great effect.

\section{Influencing factors of stable operation of biological aerated filter}

Hydraulic Load. Hydraulic load refers to the amount of water per hour of the unit area of the filter material. It is decided to the hydraulic retention time, effective volume and project investment important technical indicators of BAF. The size of the hydraulic load is directly related to the contact time of sewage with biofilm in BAF. Therefore, it is one of the significant factors that affect the treatment effect by $\mathrm{BAF}^{[7]}$. If the hydraulic load is too small, the hydraulic retention time is too long. It isn't conducive to microbial updates, and limit the improving of volume loading. Therefore, the function of BAF can't be utilized effectively, leading to increasing the investment to the project. If the hydraulic load is too large, hydraulic residence time is too short. Contacting time of sewage and microorganism will be shortened, leading to pollutants removal capacity decreased. At the same time, too strong hydraulic erosion of the filter material, will make the loss of biofilm on the filter material.

Gas-Water Ratio. It is necessary to maintain a certain amount of dissolved oxygen (DO) in the reactor in order to degrading the pollutant by microorganism in the micro-polluted water. The ratio 
can directly affect the concentration of DO in BAF. Usually, mass transfer resistance of the film will reduce with gas-water ratio increasing. Mass transfer coefficient of bubbles in the water also increase. Under similar conditions, the dissolved oxygen concentration in the biofilm increased, which resulted in the increase of the transmission and permeation rate of oxygen in the biofilm. The activity of aerobic heterotrophic bacteria and autotrophic nitrifier which can remove of organic compounds and ammonia nitrogen, also increased. Thus biological oxidation process will accelerate ${ }^{[8]}$. But the gas-water ratio can't be too large. Because of the limited solubility of oxygen, too large aeration rate can't be continued to improve DO concentration, instead of making power consumption and operating cost increase. In addition to excessive aeration, larger gas velocity produces stronger shearing force, leading to biofilm off ${ }^{[9]}$.

Packing Height. Microorganisms on the filter with bottom-up show different distribution. It is mainly due to the different kinds and quantity of the microorganism along the water flow, and the thickness of the biofilm is also different ${ }^{[7]}$. When conditions (the type and quantity of filter, filling mode, particle diameter and inflow direction) are determined, the height of the filter plays a decisive role. If the filter is not high enough, it will affect the stable operation of biological aerated filter and water quality. If the filter material is too high, it will cause economic waste and other adverse effects.

\section{Application of BAF in treatment of micro polluted water}

Japan M.Takasaki et al began the research of BAF for micro-polluted water in 80 years of the last century. The research shows: BAF was used to treat micro-polluted water. Ammonia nitrogen removal rate is about $80 \%$.even under extreme temperature conditions, the removal rate of ammonia nitrogen is in $60 \%-80 \%$. And compared with other packing processing system, BAF is not easy to be blocked ${ }^{[10]}$. Kyeong-Ho Lim from South Korea, in the 90 s of the last century, began to research treatment by BAF for drinking water, efforts to control disinfection by-products formation and improve the safety of disinfection ${ }^{[11]}$. In China, although from the 90 s of the 20th century, we began extensive research on micro-polluted water by BAF pre-processing technology. But the research process also stay in production test research stage.

Department of environmental engineering of Tsinghua University and Bengbu water plant cooperate to research pre-treatment of micro-polluted water by BAF at home for the first time with the scale of production. The engineering manly apply biological ceramic filter, processing of raw water for the Huai River. The process flow chart is shown in Fig. 1.

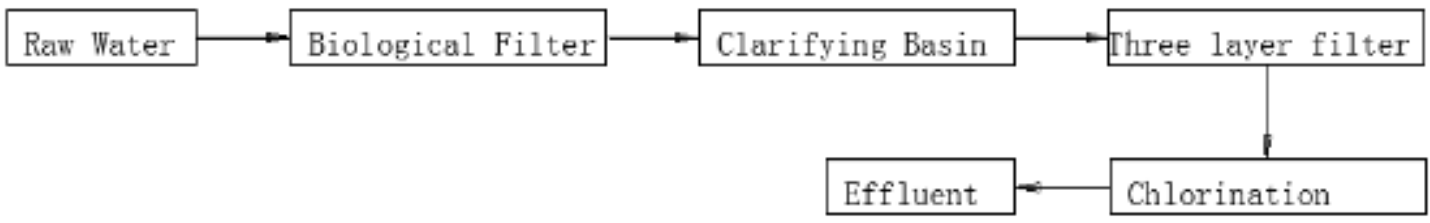

Fig.1. Treatment process of biological aerated filter

The results of the study show that biological pre-treatment in the conditions of gas water ratio of $1: 1$, the empty bed filtration velocity $3.6-6.0 \mathrm{~m} / \mathrm{h}$, organics and ammonia nitrogen removal rate were separately $13.6 \%-20.5 \%$ and $70 \%-90 \%{ }^{[12]}$.

\section{Conclusions}

Pre-treatment of micro-polluted water by BAF can effectively remove biodegradable organic compounds, reduce bacteria in the water pipe network to breed, and improve the biological stability of drinking water. The pre-treatment process, in removal of organics and ammonia, can reduce the dosage of chlorine in disinfection process and disinfection by-products. Liu et.al research showed that BAF pre-treatment can reduce the zeta potential of the colloidal material in the water, and make 
colloidal material easy to agglomerate, simultaneously reduce the dosage of coagulant and coagulant aid, thereby decreasing the treatment cost ${ }^{[13]}$.

The BAF treatment of micro-polluted water extensively studied, but the production operation of BAF is not reported in China. Only Shanghai, Bengbu, several water plant run test. The process in some aspects still need to deeply explore: the process of long-term operation stability; running effect at low temperatures; inorganic sediment, shellfish, other aquatic organisms and algae breeding on the effect of the operation ; concentration of organisms and ammonia nitrogen seasonal changes greatly.

\section{Acknowledgements}

This study was partly supported by National Natural Science Foundation of China (51278225), Science and technology development projects of Shandong province (2013GSF11704), Natural Science Foundation of Shandong province (ZR2013EEQ007, ZR2015EM021), and Science and technology development projects of Jinan (201302079).

\section{References}

[1]Jizhong Xue : China Water \&Wastewater Vol.15(1999),p 66.In Chinese.

[2]Mingshu Gong, Yunlan Yin: WATER\&WASTEWATER ENGINEERING Vol.15 (1999),p5.In Chinese.

[3]Xiaoqin Li , Yonghui Wang , Jiandong Zhou: ENVIRONMENTAL SCIENCE AND MANAGEMENT Vol.33(2008),p91.In Chinese.

[4] Xiaohui Ma, Yuhua Zhao, Jin Li: Liaoning Chemical IndustryVol.35(2009), p.714.In Chinese.

[5] Xin Liu, Xiaomin Sun, Xiaohua Bu.et al: Water Purification Technology Vol.31(2012), p.44.In Chinese.

[6]Jianhua $\mathrm{Xu}$, Hui Liu , Dong Zhang: WATER\&WASTEWATER ENGINEERING Vol.28(2002),p.1.In Chinese.

[7] XiaoMing Tan , Xiaohui Ma , Yu Bai.et al: Liaoning Chemical IndustryVol.36(2007), p.565.In Chinese.

[8] Guorong Luo, Xiaofei Sun:Ground water Vol.34(2012), p.110.In Chinese.

[9] Kangping Cui ,Shuchuan Peng, Yuanxiang Zhou: JOURNAL OF HeEFEI OF UNIVERSITY OF TECHNOLOGY Vol.4(2005),p.374.In Chinese.

[10]M. Takasak et al: Water Science \& Technology Vol.22(1990),p.137.

[11]Kyeong-Ho Lim et al: Water Science \& Technology Vol.36(1997),p.101.

[12] Wenjun Liu, Beiping He, Xihui Zhang :Environmental Science Vol.1(1997)1,p.20.In Chinese.

[13]Wenjun Liu, Beiping He, Xihui Zhang: China Water \& Wastewater Vol.12(1996),p.27.In Chinese. 